\section{Clinical Update: Conservative Management of Carpometacarpal Joint Osteoarthritis}

\section{To the Editor:}

Osteoarthritis (OA) of the thumb carpometacarpal (CMC) joint affects $15 \%$ of adults older than 30 years and $66 \%$ of women older than 55 years ${ }^{1}$. The resultant loss of thumb function may result in a $40 \%$ to $50 \%$ impairment of the upper extremity ${ }^{2}$. Thumb CMC OA is a degenerative alteration of the CMC OA joint that is characterized by abrasion, progressive deterioration of the joint surfaces, ligamentous laxity, and osteophyte formation at the site of deterioration ${ }^{3}$. Some patients with advanced CMC OA may proceed to surgical reconstruction of the basal thumb joint following failure of conservative interventions ${ }^{3}$, but it is accepted that all patients require a trial of conservative therapy before proceeding with surgery ${ }^{3}$. There are differences regarding the understanding of efficacy of conservative interventions among orthopedic specialists, rheumatologists, and hand therapists. It is important that specialists understand which interventions have been found to be the most efficacious to improve the individual's quality of life $\mathrm{e}^{4}$.

The goals of conservative treatment are predominately 2-fold: relief of pain, and improvement of hand function. Therapeutic interventions for patients with $\mathrm{OA}$ of the hand can include joint-protection technique instruction, manual therapy, adaptive equipment provision and instruction, heat modalities, orthotic provision, strengthening and range-of-motion exercises, adaptive technique instruction, patient education in symptom control techniques, and provision of a home exercise program ${ }^{4}$. There have been 2 recent systematic reviews that evaluated the quality of the evidence for the conservative management of $\mathrm{CMC} \mathrm{OA}^{5,6}$. The authors of one of the reviews ${ }^{5}$ used the Physiotherapy Evidence Database (PEDro) scale to assess the methodological quality of the studies included in the review. The PEDro scores of the studies ranged from 3 to 10 . The reviews both cite the need for additional high-quality research on the best available conservative treatment for patients with CMC OA to improve the quality of care for this large patient population ${ }^{5,6}$.

In early $\mathrm{OA}$, the focus of conservative management should emphasize reduction of pain and improvement of functional capacities ${ }^{7}$. Further, prevention of progression of joint damage and improvement of quality of life should also be addressed. There are nonpharmacological and pharmacological conservative treatment interventions. In many patients, these modalities are combined and tailored to address individual needs and risk factors throughout the disease process ${ }^{8}$. In longterm disease management, patient education is thought to be effective and necessary to increase the compliance of patients, although there are few reported benefits 9 . Symptoms can be reduced by providing the patient with information about OA, its symptoms, the objectives of its treatment, and the importance of changes in lifestyle - although the effect size of these interventions is small $(<0.20)^{10,11}$. The European League Against Rheumatism has published evidence-based guidelines for the treatment of hand $\mathrm{OA}^{12}$.

There is recent evidence that conservative interventions performed in therapy may improve hand function and decrease hand pain in patients with $\mathrm{CMC} \mathrm{OA}^{5}$. There is moderate quality evidence that manual therapy and therapeutic exercise improves pain in patients with thumb CMC OA at both short- and intermediate-term followup ${ }^{5}$. There is some evidence that both steroid and hyaluronate intraarticular injections can reduce pain ${ }^{6}$, and low to moderate quality evidence that magnetic therapy improves pain and function at short-term followup (effect size for pain $-1.39,95 \%$ CI $-2.00--0.78)^{5}$. The effect of manual therapy and therapeutic exercise had a large effect size of -116.67 , with a $95 \%$ CI of $-137.55--95.78$ for the reduction of pain ${ }^{13}$. Our study demonstrated a large treatment effect with a CI that did not cross 0 . The effect size of orthoses (splints) on pain reduction was small and not significant $(\mathrm{g}=0.46)$ with a range from $-1.08-0.16$ at a short-term followup, while it was very large but not significant $(\mathrm{g}=1.65)$ with a range from $-4.04-0.74$ at a longterm followup ${ }^{5}$. Manual therapy was effective in the reduction of pain at the short-term followup (effect size of $g=-1.86$ ), with a range of $-3.700--0.025$. There is very low to low quality evidence that other conservative interventions provide no significant improvement in pain or function at short- and longterm followup. Commonly used treatment modalities are lasers, ultrasound, electrotherapy, or acupuncture, but high-quality clinical trials investigating these modalities are scarce ${ }^{5}$.

Some of the commonly used conservative interventions performed in therapy have varied levels of evidence to support their use to improve hand function and decrease hand pain. Additional randomized controlled trials are needed to support interventions that resolve emerging evidence regarding thumb proprioception deficits and kinematic alterations. Successful management of the thumb CMC OA requires a collaborative effort from rheumatologists, orthopedic specialists, therapists, and patients. Such working relationships should allow optimum preservation of thumb function over time.

JORGE HUGO VILLAFAÑE, PhD, MSc, PT, Researcher, Istituto di Ricovero e Cura a Carattere Scientifico (IRCCS) Don Gnocchi Foundation, Milan, Italy; KRISTIN VALDES, OTD, OT, CHT, Drexel University, Philadelphia, Pennsylvania, USA; PEDRO BERJANO, MD, PhD, IRCCS Istituto Ortopedico Galeazzi, Milan, Italy; ANNE WAJON, $\mathrm{PhD}, \mathrm{MAppSc}(\mathrm{Phty}), \mathrm{CHT}$, Macquarie Hand Therapy, Sydney, Australia. Address correspondence to Dr. J.H. Villafañe, Regione Generala 11/16, Piossasco 10045, Italy. E-mail: mail@villafane.it

\section{REFERENCES}

1. Haara MM, Heliovaara M, Kroger H, Arokoski JP, Manninen P, Kärkkäinen A, et al. Osteoarthritis in the carpometacarpal joint of the thumb. Prevalence and associations with disability and mortality. J Bone Joint Surg Am 2004;86:1452-7.

2. Luker KR, Aguinaldo A, Kenney D, Cahill-Rowley K, Ladd AL. Functional task kinematics of the thumb carpometacarpal joint. Clin Orthop Relat Res 2014;472:1123-9.

3. Matullo KS, Ilyas A, Thoder JJ. CMC arthroplasty of the thumb: a review. Hand 2007;2:232-9.

4. Valdes K, Marik T. A systematic review of conservative interventions for osteoarthritis of the hand. J Hand Ther 2010;23:334-50.

5. Bertozzi L, Valdes K, Vanti C, Negrini S, Pillastrini P, Villafañe JH Investigation of the effect of conservative interventions in thumb carpometacarpal osteoarthritis: systematic review and meta-analysis. Disabil Rehabil 2015 Jan 5 (E-pub ahead of print).

6. Spaans AJ, van Minnen LP, Kon M, Schuurman AH, Schreuders AR, Vermeulen GM. Conservative treatment of thumb base osteoarthritis: a systematic review. J Hand Surg Am 2015;40:16-21

7. Wesseling J, Dekker J, van den Berg WB, Bierma-Zeinstra SM, Boers M, Cats HA, et al. CHECK (Cohort Hip and Cohort Knee) similarities and differences with the Osteoarthritis Initiative. Ann Rheum Dis 2009;68:1413-9.

8. Bijlsma JW, Berenbaum F, Lafeber FP. Osteoarthritis: an update with relevance for clinical practice. Lancet 2011;377:2115-26.

9. May S. Self-management of chronic low back pain and osteoarthritis. Nat Rev Rheumatol 2010;6:199-209.

10. Zhang W, Moskowitz RW, Nuki G, Abramson S, Altman RD, Arden $\mathrm{N}$, et al. OARSI recommendations for the management of hip and knee osteoarthritis, Part II: OARSI evidence-based, expert consensus guidelines. Osteoarthritis Cartilage 2008;16:137-62.

11. Zhang W, Nuki G, Moskowitz RW, Abramson S, Altman RD, Arden $\mathrm{NK}$, et al. OARSI recommendations for the management of hip and knee osteoarthritis: part III: Changes in evidence following systematic cumulative update of research published through January 2009. Osteoarthritis Cartilage 2010;18:476-99.

12. Zhang W, Doherty M, Leeb BF, Alekseeva L, Arden NK, Bijlsma JW, et al. EULAR evidence based recommendations for the management of hand osteoarthritis: report of a Task Force of the EULAR Standing Committee for International Clinical Studies Including Therapeutics (ESCISIT). Ann Rheum Dis 2007; 66:377-88. 
13. Villafañe JH, Cleland JA, Fernández-de-Las-Peñas C. The effectiveness of a manual therapy and exercise protocol in patients with thumb carpometacarpal osteoarthritis: a randomized controlled trial. J Orthop Sports Phys Ther 2013;43:204-13.

J Rheumatol 2015;42:9; doi:10.3899/jrheum.150416 